\title{
Self-Powered Triboelectric Micro Liquid/Gas Flow Sensor for Microfluidics
}

Jie Chen, ${ }^{\dagger,},{ }^{\perp}$ Hengyu Guo, ${ }^{\dagger} \perp$ Jiangeng Zheng, ${ }^{\dagger}$ Yingzhou Huang, ${ }^{\dagger}$ Guanlin Liu, ${ }^{\dagger}$ Chenguo Hu, ${ }^{\dagger, *}$ Zhong Lin Wang ${ }^{\S, *}$

${ }^{\dagger}$ Department of Applied Physics, Chongqing University, Chongqing 400044, China

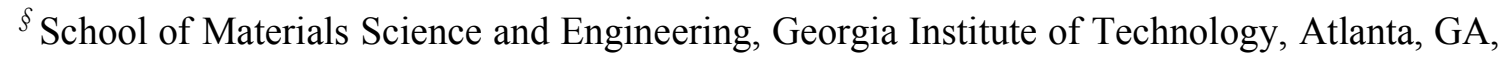
30332-0245, United States.

${ }^{\perp}$ These authors contributed equally to this work. The author order was determined by coin toss.

*Correspondence to: hucg@cqu.edu.cn; zhong.wang@mse.gatech.edu;

KEYWORDS: Micro-fluidic sensor, triboelectric, self-powered system. 


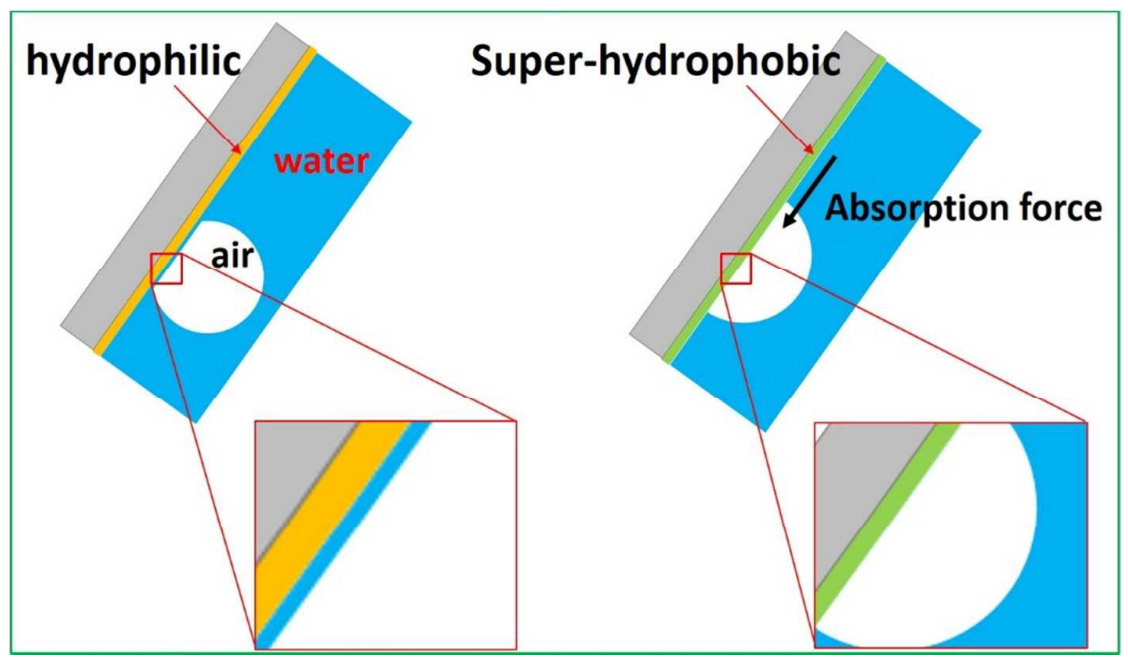

Supporting Information S1. When the tribo-layer is hydrophilic, the air bubble can go through easily, however, there will be a water layer between the tribolayer and air bubble. When the tribolayer is super-hydrophobic, the air bubble can be absorbed on the tribolayer. 


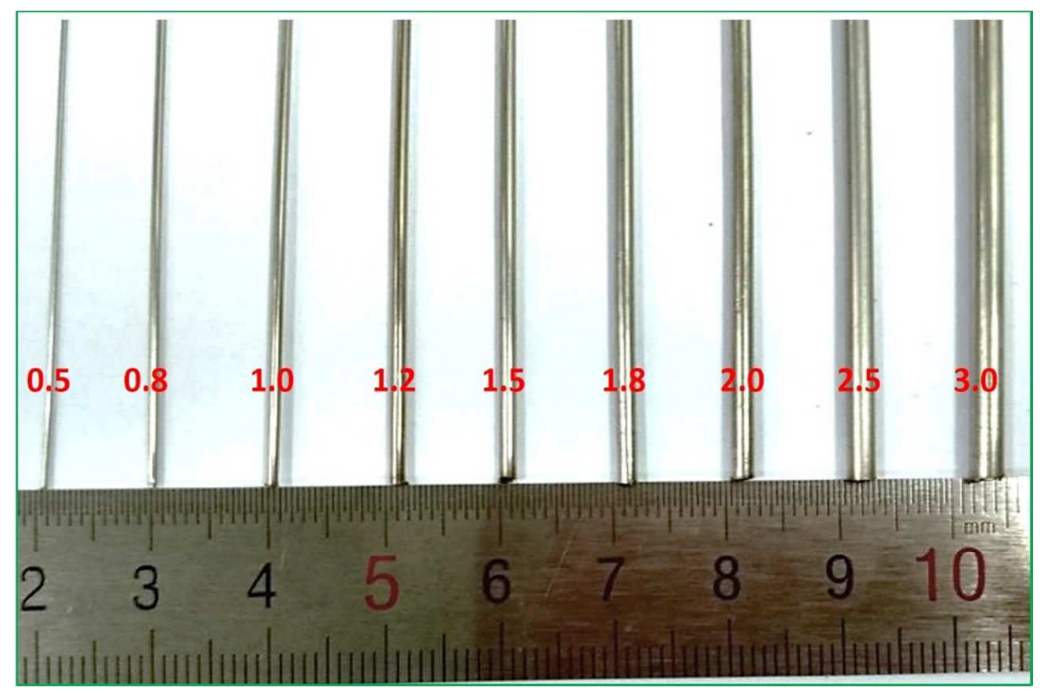

Supporting Information S2. The digital photography of steel capillaries from 0.5 to $3 \mathrm{~mm}$ diameter. 


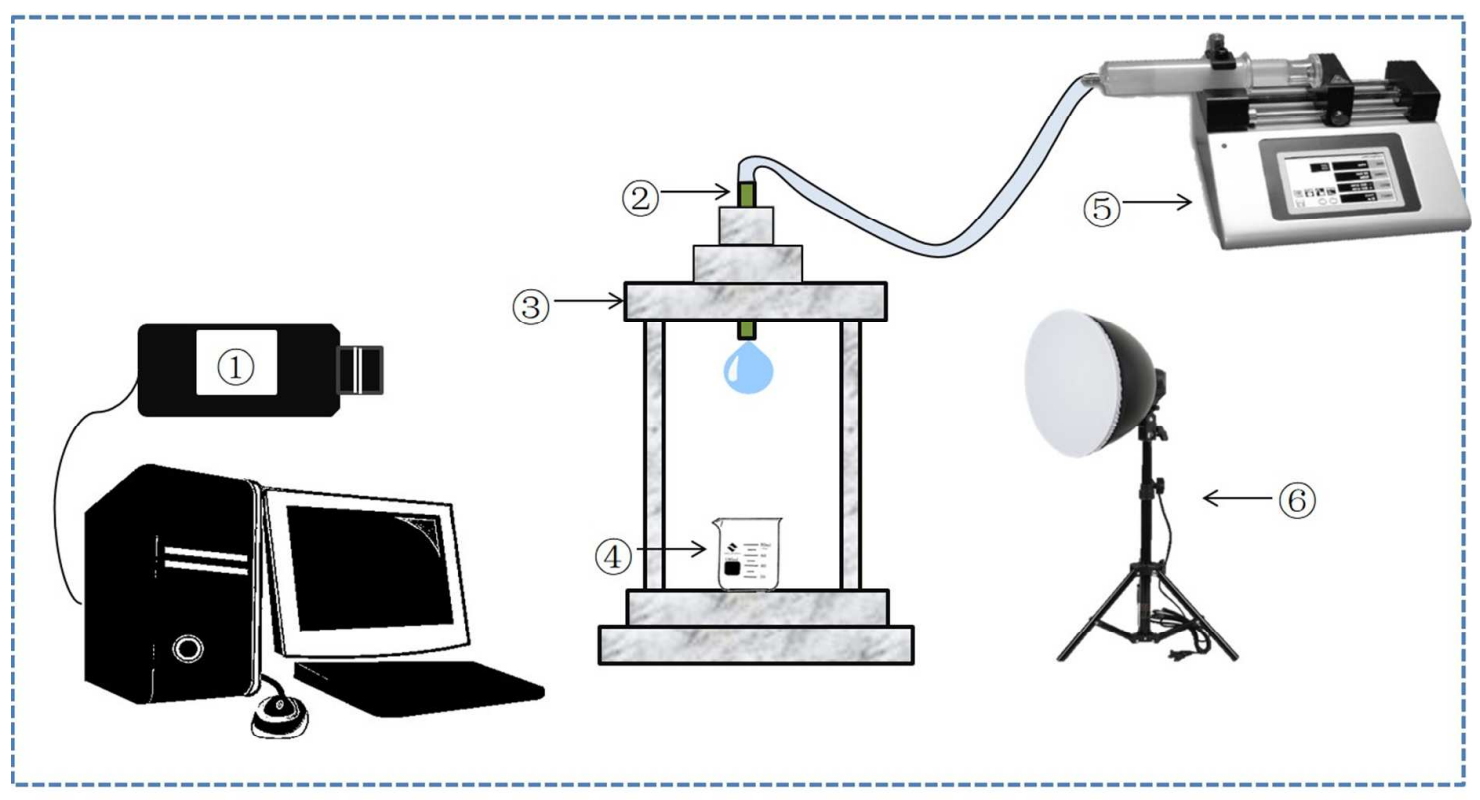
(1) high speed camera
(4) beaker
(2) steel capillary
(5) micro-pump
(3) acrylic frame
(6) light source

Supporting Information S3. Sketch of apparatus for measuring the water droplets. 


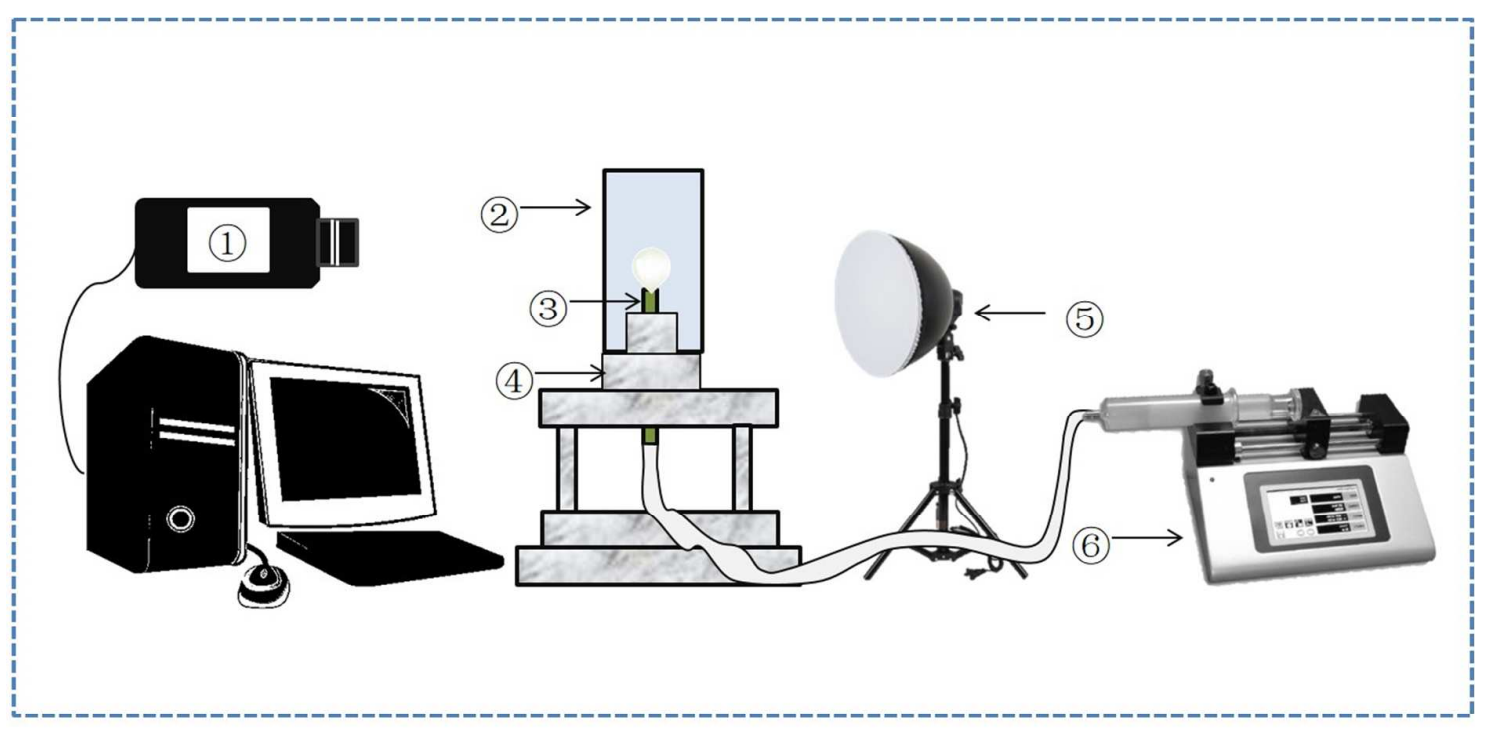
(1) high speed camera
(4) acrylic frame
(2) glass column
(5) light source
(3) steel capillary
(6) micro-pump

Supporting Information S4. Schematic diagram of the experimental facility for measuring the air bubbles. 


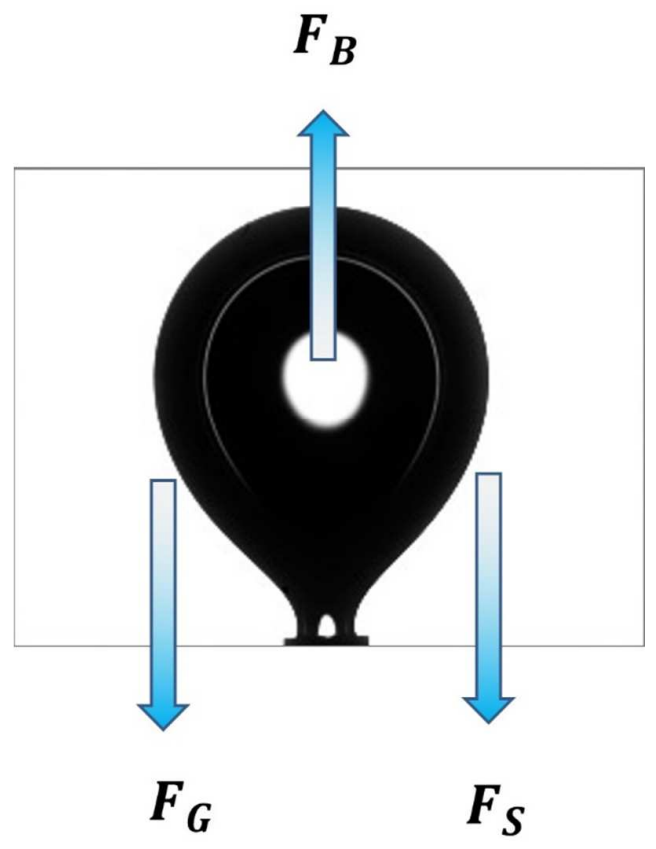

Supporting Information S5. Schematic diagram of the vertical forces on a bubble during formation from a capillary tip. 

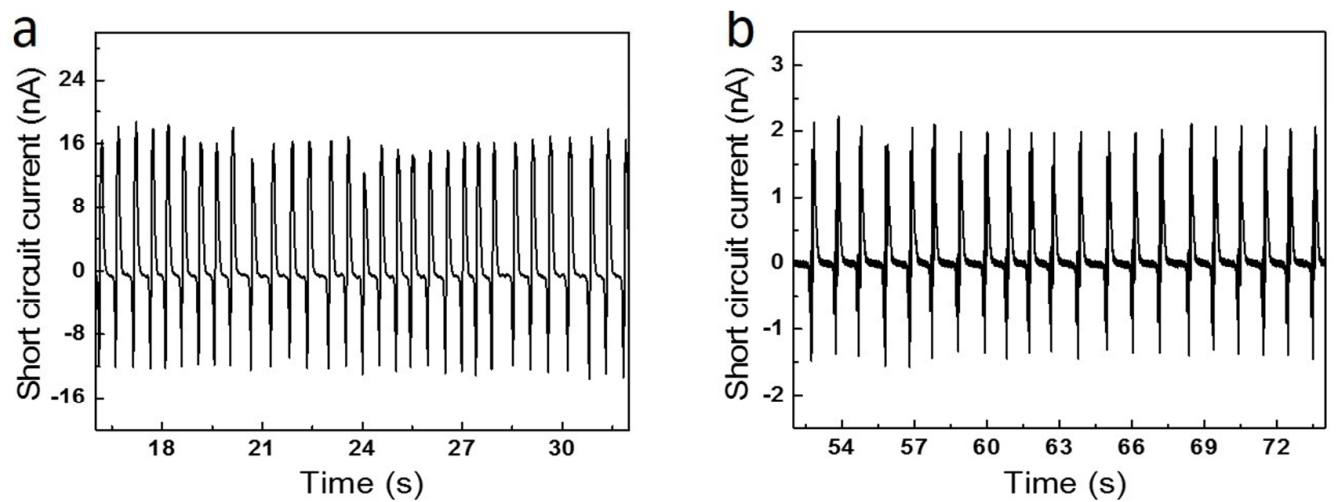

Supporting Information S6. (a) The output current signal of liquid flow sensor. (b) The output current signal of gas flow sensor. 

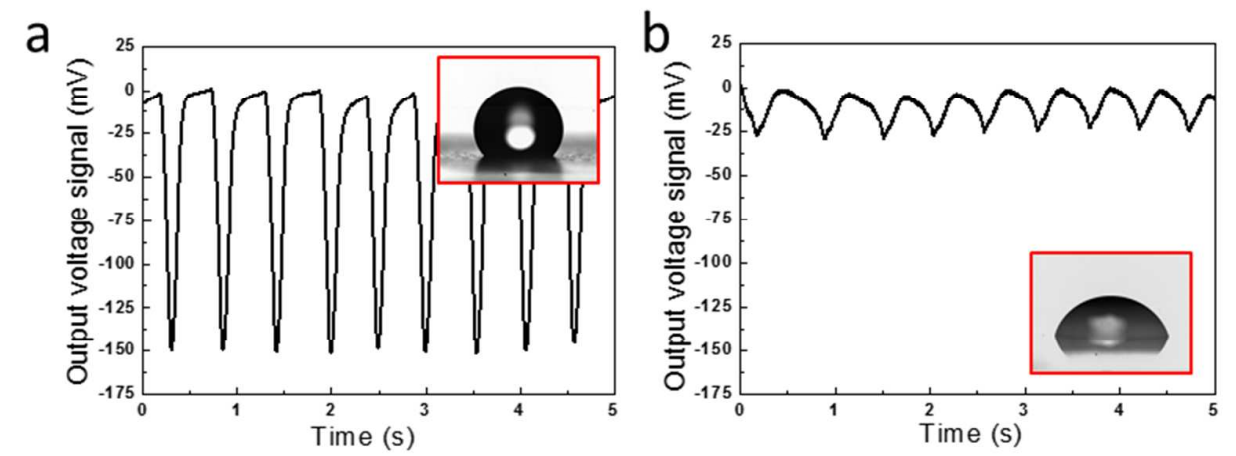

Supporting Information S7. The output voltage signal of the Liquid-TMS when using PTFE (a) and Kapton (b) as tribo-layer. 


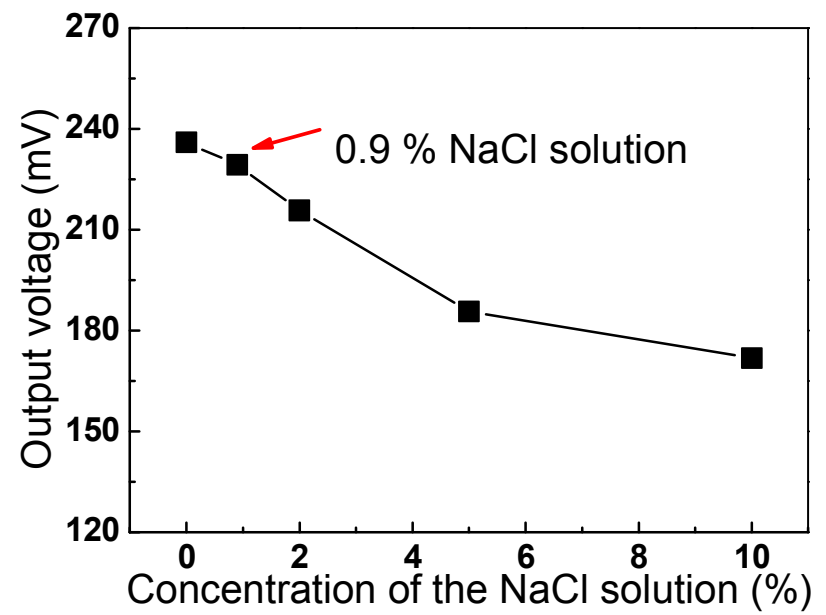

Supporting Information S8. The output amplitude of the Liquid-TMS when using different concentration of $\mathrm{NaCl}$ aqueous solution. 

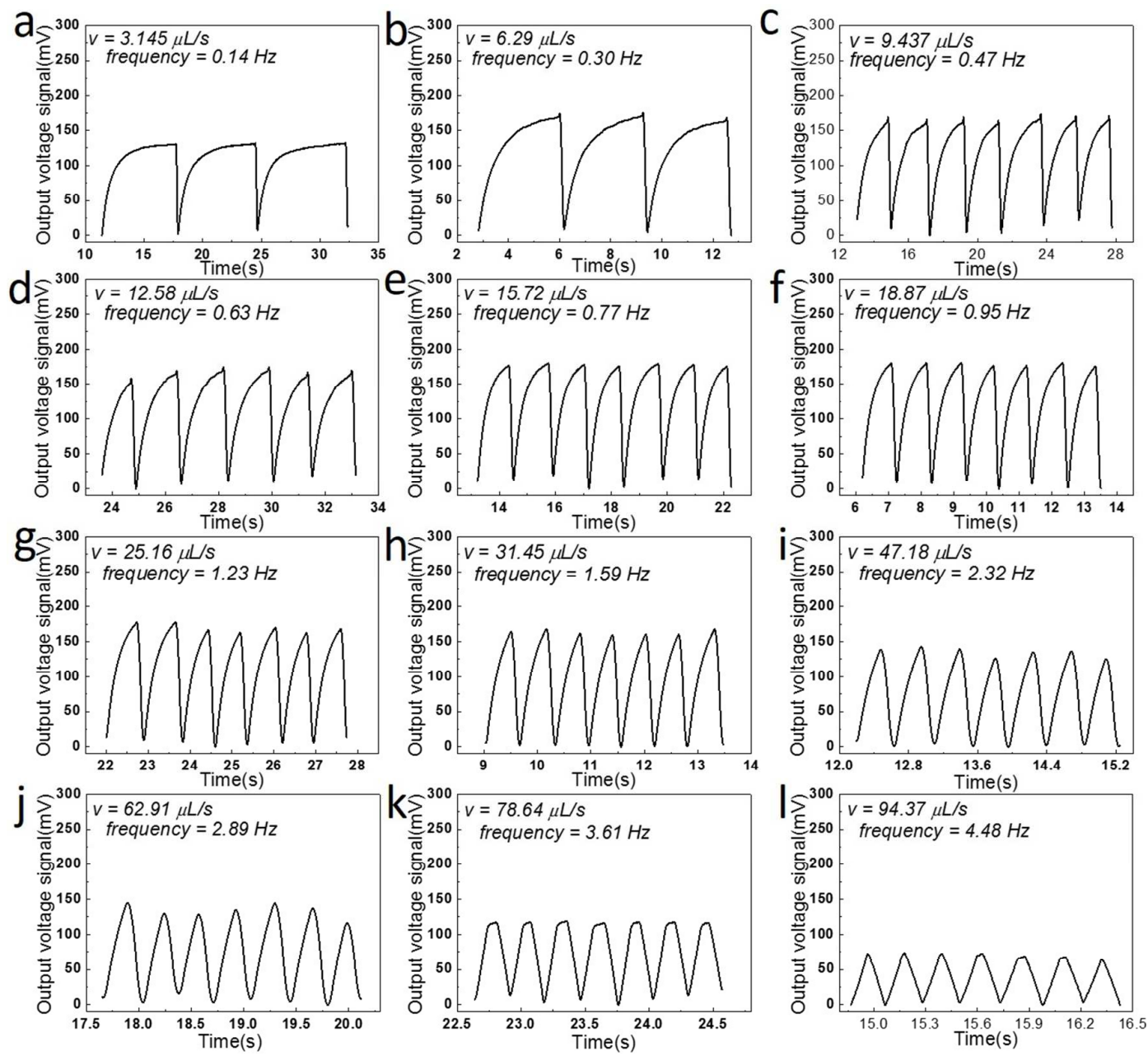

Supporting Information S9. (a)-(1) The output voltage curve of Liquid-TMS under various external flow rate. 

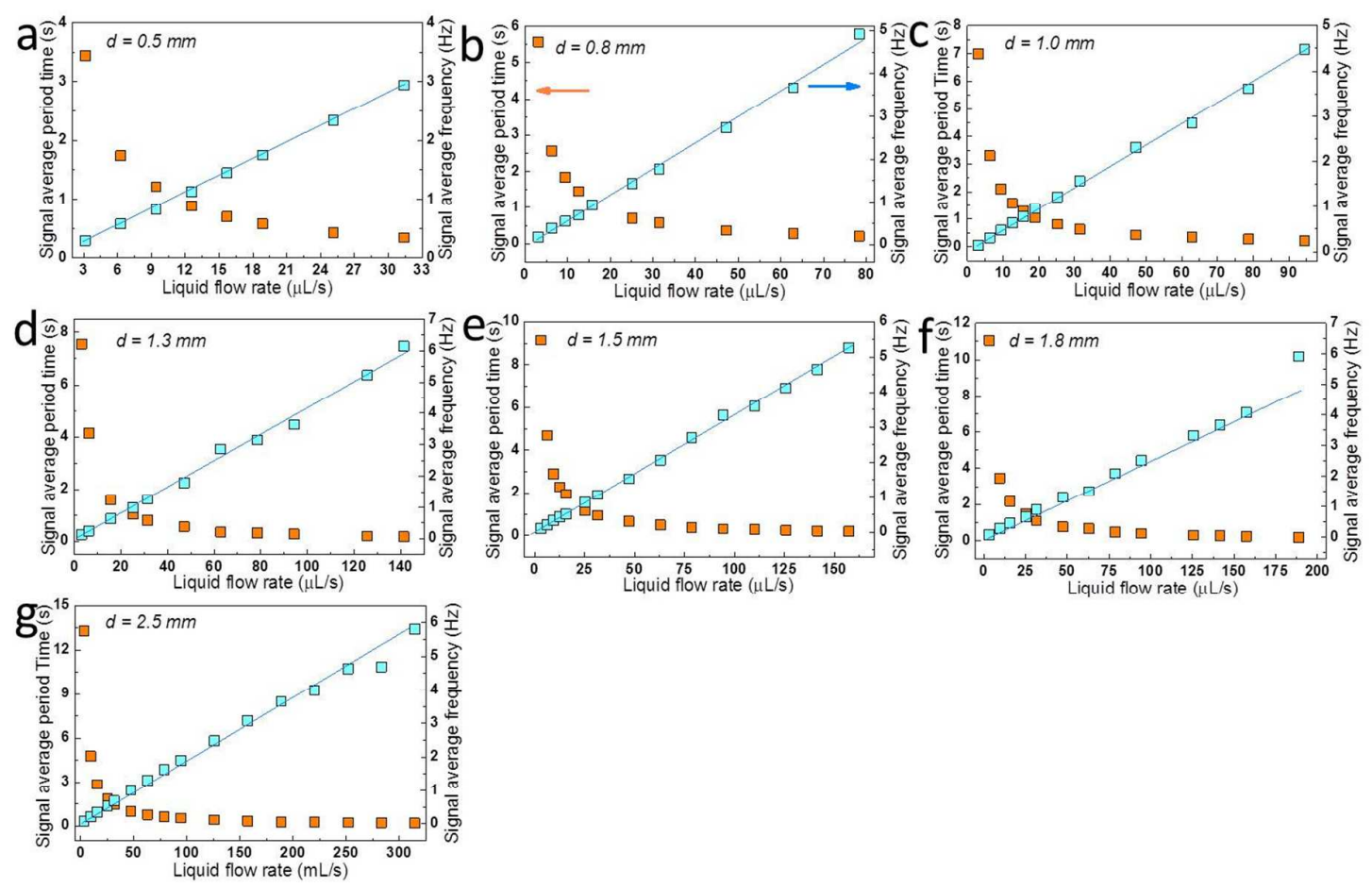

Supporting Information S10. (a)-(g) Dependence of the signal average period time/frequency on liquid flow rate measured at $0.5,0.8,1.0,1.3,1.5,1.8,2.5 \mathrm{~mm}$ capillaries. 

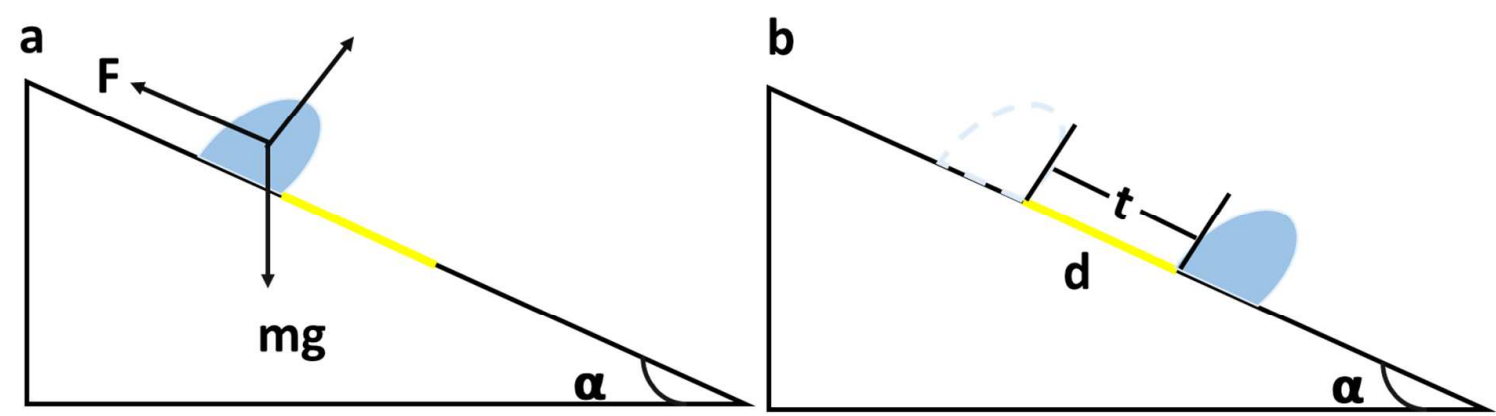

Supporting Information S11. (a) Schematic diagram of the forces on a droplet during the movement process in the inner wall of PE tube. (b) Schematic diagram of the droplet's initial state (just contact with electrode) and final state (separation with electrode).

The time $t$ of each droplet passing through the electrode can be calculated by

$$
\begin{aligned}
& {[m g \sin \alpha-F(\alpha)] d=\frac{1}{2} m v^{2}-\frac{1}{2} m v_{0}(h)^{2}} \\
& {[m g \sin \alpha-F(\alpha)] t=m v-m v_{0}(h)} \\
& F=S A(\alpha)
\end{aligned}
$$

Where $\alpha$ is the tilt angle, $F$ is the adhesion force which depends on the contact area $A(\alpha)$ and the coefficient of adhesion $S, d$ is the length of electrode, $v$ is the final velocity when the droplet pass through the electrode, $v_{0}(h)$ is the initial velocity related to the height.

According to eq. (1)- (3), the time $t$ can be expressed by

$$
t=\frac{\sqrt{2 m[m g \sin \alpha-S A(\alpha)] d+m^{2} v_{0}^{2}}-m v_{0}}{[m g \sin \alpha-S A(\alpha)] d}
$$

When the initial velocity is zero, eq. (4) can be expressed in simple form

$$
t=\sqrt{\frac{2 m}{[m g \sin \alpha-S A(\alpha)] d}}
$$

It proves that the tilt angle can affect the time $t$ of each droplet passing through the electrode. And the interval time of two output signal peaks is related to $t$. Especially, when the next droplet is well-formed at the moment of the former passing through the electrode, the interval time is equal to $t$. But, if the growth time of each droplet is less than $t$, the next signal is indistinguishable. Therefore, the tilt angle $\alpha$ can affect the detecting range. 


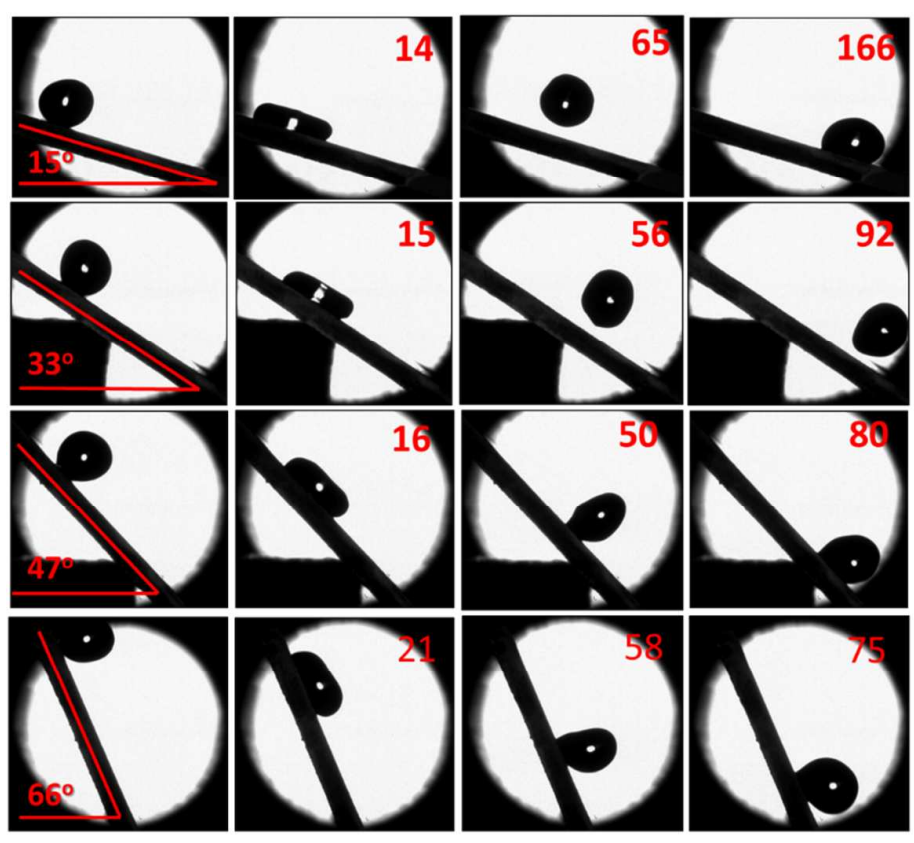

Supporting Information S12. The photography of the droplet formed by $1 \mathrm{~mm}$ capillary passing through the electrode at different tilt angle $\left(15^{0}, 33^{0}, 47^{0}, 66^{\circ}\right)$. The corresponding number on the picture is frames and the acquisition rate is 3000 frames per second. 

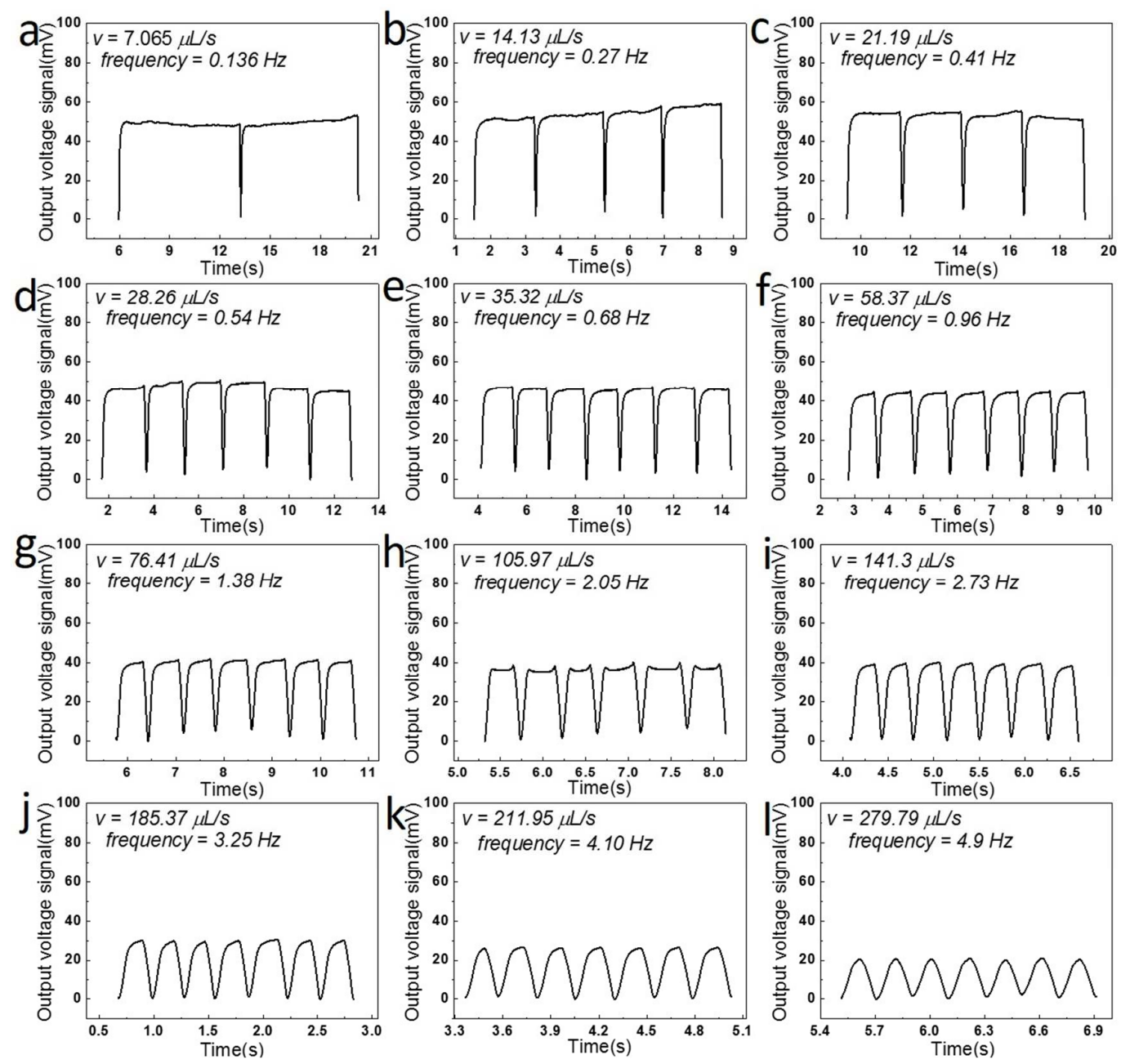

Supporting Information S13. (a)-(1) The output voltage curve of Gas-TMS under various external flow rate. 

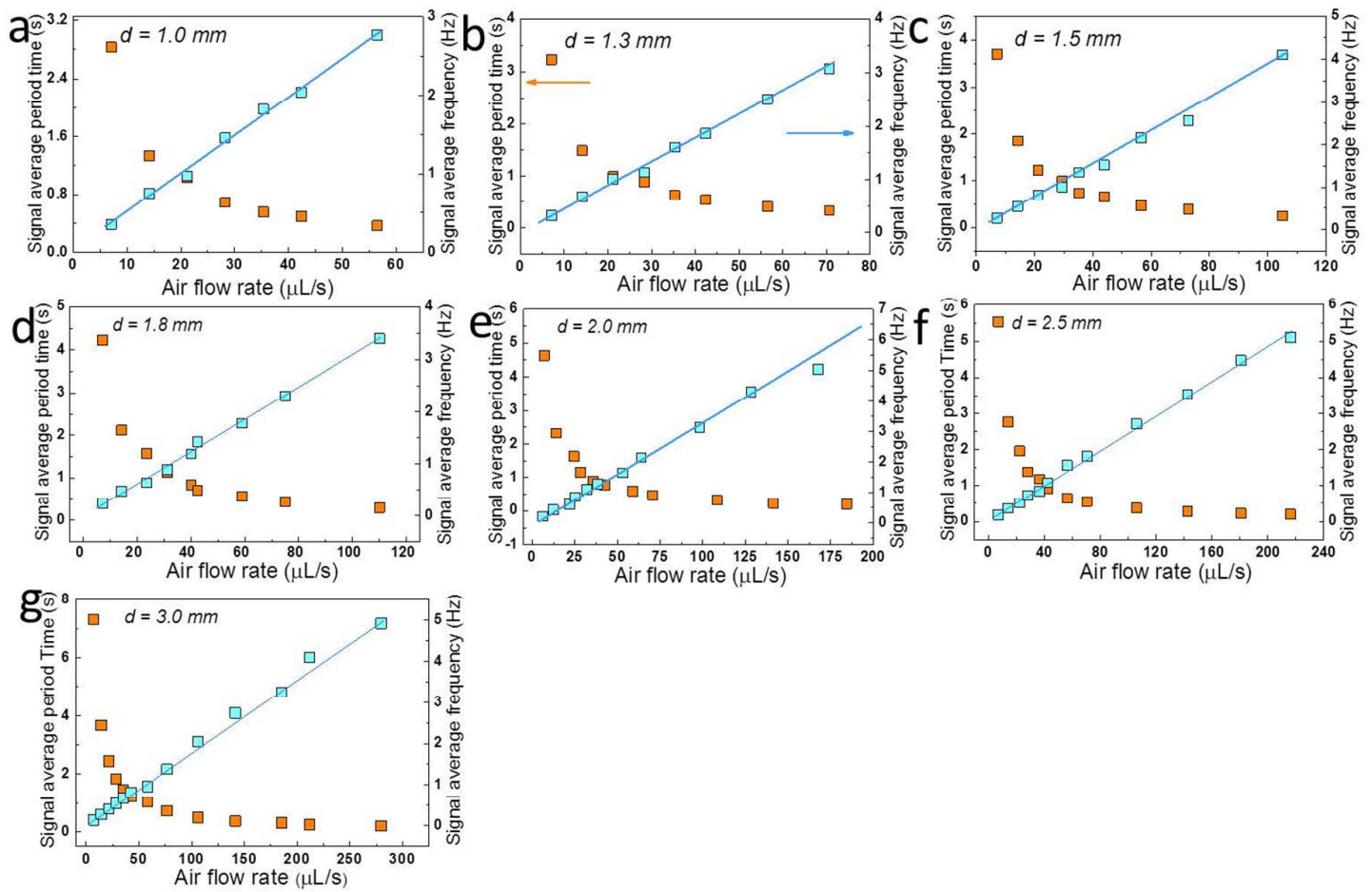

Supporting Information S14. (a)-(g) Dependence of the signal average period time/frequency on gas flow rate measured at 1.0, 1.3, 1.5, 1.8, 2.0, 2.5, $3.0 \mathrm{~mm}$ capillaries. 